\title{
Cellular distribution of human leucocyte adhesion molecule ICAM-3
}

J L Cordell, K Pulford, H Turley, M Jones, K Micklem, I A Doussis, X Tyler, K Mayne, K C Gatter, D Y Mason

\begin{abstract}
Aims-To describe the distribution of the recently cloned human leucocyte adhesion molecule ICAM-3 in normal and neoplastic tissues and cell lines.

Methods-A panel of four monoclonal antibodies to ICAM-3 were used to stain cell lines and sections of human lymphoid tissues using the alkaline phosphatase-anti-alkaline phosphatase immunocytochemical method (APAAP).

Results-In peripheral blood ICAM-3 was detected on monocytes, granulocytes, and most lymphocytes. In sections of human lymphoid tissue the antigen was also found on most lymphocytes, but many of the proliferating $B$ cells found in the germinal centres of secondary lymphoid follicles were ICAM-3 negative. ICAM-3 was also found on neoplastic white cells (in chronic lymphocytic leukaemia, hairy cell leukaemia, acute and chronic myeloid leukaemia, and multiple myeloma) with the exception of Reed-Sternberg cells in Hodgkin's disease, many of which were negative. ICAM-3 was consistently absent from cells and tissues of non-haemopoietic origin. Endothelium (which expresses ICAM-1) was negative for ICAM-3, with the exception of vessels in some neoplastic lymphoid samples which showed variable staining for ICAM-3.

Conclusions-These findings suggest that ICAM-3 is essentially restricted to the haemopoietic system and is reciprocal in its expression to ICAM-1, in that it is present on resting cells and its level falls as a result of cell activation.
\end{abstract}

(F Clin Pathol 1994;47:143-147)

Leukaemia Research

Immunodiagnostics

Unit, Nuffield

Department of

Pathology, John

Radcliffe Hospital,

Oxford, OX3 9DU

$\mathrm{J} L$ Cordell

K Pulford

H Turley

M Jones

K Micklem

I A Doussis

$\mathrm{X}$ Tyler

K Mayne

K C Gatter

D Y Mason

Correspondence to: Jacqueline L Cordell

Accepted for publication

21 September 1993 Lymphocyte function-associated molecule 1 (LFA-1) is a leucocyte integrin which has a major role in cell adhesion. ${ }^{12}$ It mediates a wide range of leucocyte interactions in immune and inflammatory responses and acts as a receptor for the intercellular adhesion molecules ICAM-1 (CD54) and ICAM-2.3-6 It has been suggested that a third form for ICAM exists ${ }^{78}$ and recently Fawcett et $a l^{9}$ and Vazeux et al $^{10}$ described the molecular cloning of such a gene (designated ICAM-3), the product of which is also a ligand for LFA-1.
Methods

Four monoclonal antibodies (designated By44, CG106, KS128, KS118) with identical pan leucocyte reaction patterns have been identified in this laboratory over the past 10 years. These antibodies came from experiments in which murine hybridomas were prepared using standard techniques, ${ }^{11}$ following immunisation with fresh B cell lymphoma cells (antibody By44), fresh T cell leukaemia cells (antibody CG106) or Karpas 299, a T cell line with a 2:5 translocation (KS128 and KS118). Initial screening was carried out on peripheral blood smears containing immunising cells (By44 and CG106) or on cryostat sections of lymph node affected by Hodgkin's disease (KS118 and KS128). Further screening was performed using normal blood smears and cryostat sections of normal tonsil.

Normal peripheral blood mononuclear cells were separated on Lymphoprep (Nyegaard, Oslo) and cytocentrifuge preparations were made. All slides were fixed and stored frozen as described before. ${ }^{12}$ Tissues were snap frozen in liquid nitrogen, and cryostat sections $(8 \mu \mathrm{m})$ were cut, fixed, and stored as described before. ${ }^{13}$

All cell lines were cultured at $37^{\circ} \mathrm{C}$ in RPMI 1640 medium containing 10\% fetal calf serum (FCS) (Gibco Biocult Ltd). Cytocentrifuge preparations were made and treated as described before. To promote their differentiation towards macrophages U937 and HL60 cells were incubated with $1.6 \times$ $10^{-8} \mathrm{M}$ 12-0-tetradecanoylphorbol 13-acetate (TPA, Sigma Chemical Co) in RPMI 1640 containing $10 \%$ FCS for up to 12 days.

Antibodies against CD3, CD10, CD11b, CD19, CD68 and cytokeratin were obtained from DAKO a/s (Glostrup, Denmark). Antibodies to ICAM-1 (RR1/1) and ICAM-2 (CBR-IC2/1) were provided by Dr TA Springer.

The APAAP staining technique was carried out as described before.1213 The enzyme reaction was developed using naphthol AS-MX phosphate and Fast Red (TR-Salt, Sigma Chemical (Co) as substrate.

Immunoperoxidase staining was performed as follows: tissue sections were incubated first with a monoclonal antibody for 30 minutes, followed by peroxidase conjugated rabbit antimouse immunoglobulin (DAKO, a/s). The peroxidase reaction was developed using diaminobenzidine-hydrogen peroxide substrate. ${ }^{14}$ 
Figure 1 (A) Staining of WOP cell line after transfection with an ICAM-3 cDNA clone, using the anti-ICAM-3 antibody By44. Numerous positive cells are seen. Inset: cells transfected with control cDNA, stained under the same conditions. (B-D) Staining of reactive lymphoid tissue (tonsil) with antibodies to ICAM-1, 2 and 3. (B) ICAM-1 is strongly expressed on germinal centre (GC) cells and also on vascular endothelium (arrowed) in the T cell area. (C) ICAM-2 is absent from the germinal centre but is present on vascular endothelium (arrowed). (D) ICAM-3 is expressed on many cells in the extrafollicular $T$ cell area and on $B$ cells in the mantle zone, but many cells in the germinal centre are negative. Vascular endothelium is negative. $(E-G)$ Staining of human spleen with antibodies to ICAM-1, 2 and 3. (E) ICAM-1 is seen on cells in the red pulp $(R P)$ and also on vascular endothelium (arrowed) and connective tissue. (F) ICAM-2 is restricted to vascular endothelium

(arrowed). Inset: Vascular endothelium at higher power. (G) ICAM-3 is not seen in the red pulp or on endothelial cells but is present on white cells lying within a vessel (arrowed). The inset shows this reactivity at higher power.

(H-F) Human kidney stained with antibodies to ICAM-1, 2, and 3. (H) ICAM-1 is strongly expressed on glomeruli (arrowed). These

structures are weakly stained for ICAM-2 (I)

and unlabelled for ICAM3 (F).

$(K-M)$ Staining of $a$ section from a case of multiple myeloma for ICAM-3. (K) Negative control. (L) Strong expression of ICAM-3 on neoplastic cells and also on vascular endothelium, seen at higher power in $(M)$.

(N) Immunostaining for

ICAM-3 in a case of

Hodgkin's disease showing strong staining of infiltrating lymphoid cells but weak or negative staining of Reed-Sternberg cells (seen in higher magnification below). All staining was performed on cryostat sections using the APAAP immunoalkaline

phosphatase technique, with the exception of $(A)$ in which staining was performed by the immunoperoxidase technique on acetone fixed cytospin preparations.

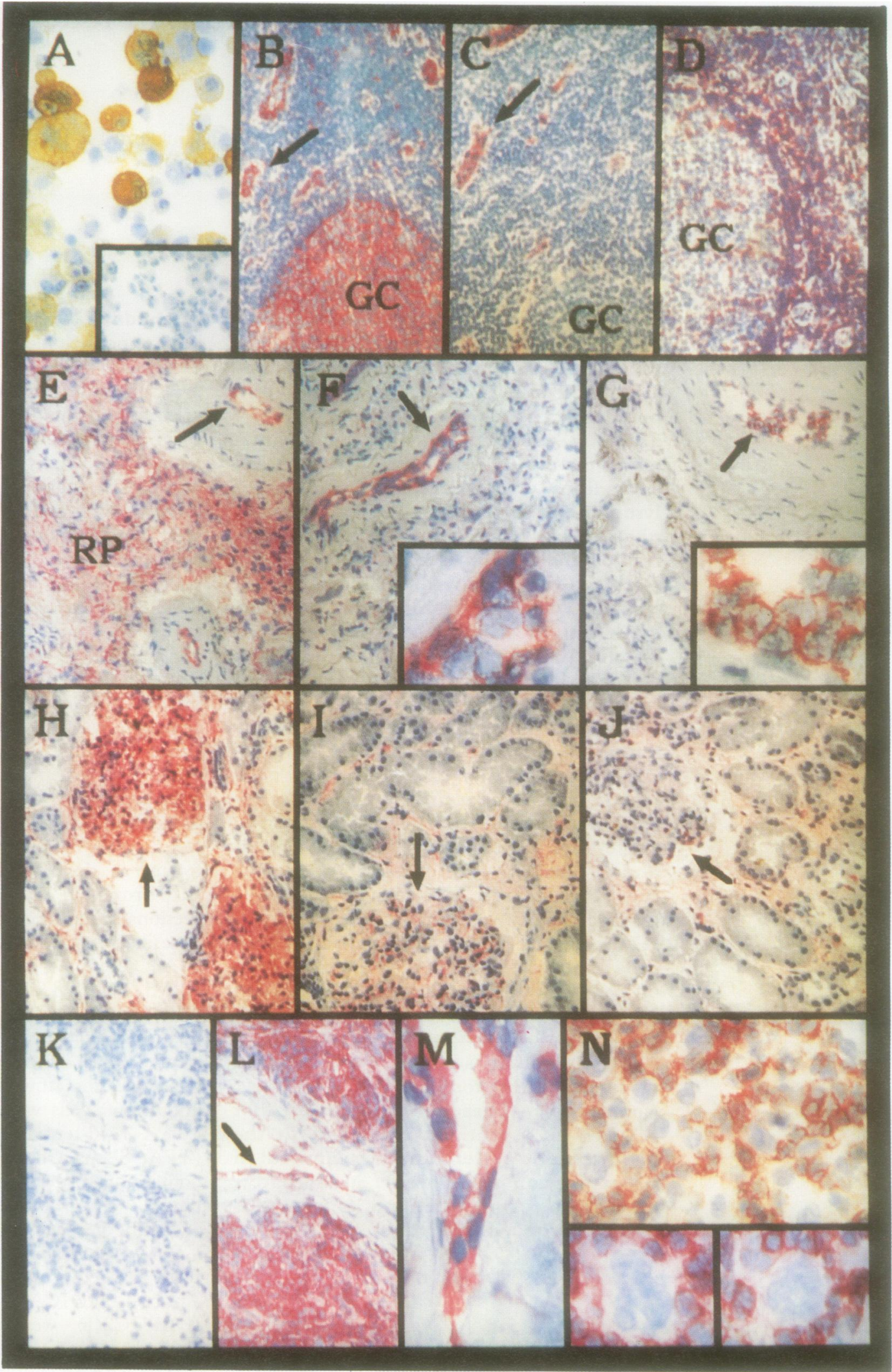

For the FACS analysis, mononuclear cell suspensions were prepared as described above, and leucocytes were isolated from whole blood by red cell lysis using ammonium chloride. ${ }^{15}$ After washing, the cells were incubated for 30 minutes at $4^{\circ} \mathrm{C}$ with monoclonal antibody. After a further wash the cells were incubated for 30 minutes with fluorescein isothiocyanate (FITC) conjugated goat antimouse $\mathrm{F}\left(\mathrm{ab}^{\prime}\right)_{2}$ immunoglobulin (DAKO a/s). The cells were then washed, fixed in $1.5 \%$ formaldehyde in phosphate buffered saline (PBS), and analysed in a FACScan flow cytometer (Becton Dickinson) using Consort 30 software.

In some experiments cells were double labelled by incubation for 30 minutes with FITC conjugated By44, prepared as described by Goding ${ }^{16}$ and then, after washing, the cells were incubated with phycoerythrin (PE)-conjugated antibody against CD3, After a final wash the cells were fixed and analysed in the FACScan flow cytometer, CD4, CD8, CD10 or CD19 (DAKO a/s). 


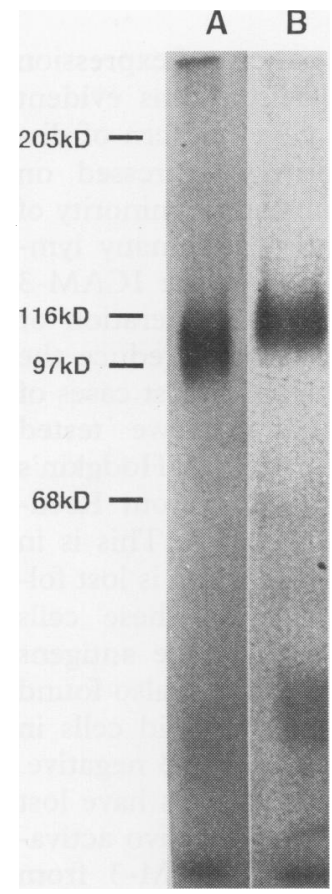

Figure 2

Immunopreciptation of ICAM-3 from surface ${ }^{125 I}$ labelled chronic

lymphocytic leukaemia cells with antibody By44 shows $a$ band of 104 kilodaltons, unreduced (lane $A$ ) and 110 kilodaltons, reduced lane B). Positions of molecular weight standards are indicated. using two colour fluorescence and Paint-aGate software.

For the cell transfection experiment, we used a 1.7 kilobase cDNA insert which contains the ICAM-3 gene. This was isolated as described before. ${ }^{9}$ COS cells were transfected with this cDNA. ${ }^{17}$ Cytospin preparations were prepared, fixed, and stored as described before, and stained using the immunoperoxidase technique. All four antibodies stained cells transfected with this clone (fig 1).

For the immunoprecipitation, leucocytes prepared from a case of chronic lymphocytic leukaemia were surface labelled with $1 \mathrm{~m} \mathrm{Ci}$ ${ }^{125}$ I iodide (Amersham) in the presence of $20 \mu$ g Iodogen (Pierce). The cells were lysed in buffer containing $2 \%$ Nonidet P40 and protease inhibitors. The lysate material was precleared using affinity purified rabbit antimouse immunoglobulin (DAKO a/s) bound to Staphylococcus A (Staph A, Sigma). Monoclonal antibodies were added to the precleared lysate and after one hour, rabbit antimouse immunoglobulin bound to Staph A was added. After a further hour the Staph A was washed in buffer containing $1 \%$ NP40 and $150 \mathrm{mM}$ sodium chloride. Immunoprecipitates were analysed by sodium dodecyl sulphate polyacrylamide gel electrophoresis (SDS-PAGE) followed by autoradiography of the dried gel using an enhancing screen. ${ }^{18}$

All four antibodies immunoprecipitated an antigen with a molecular weight of 104 kilodaltons in the unreduced state and 110 kilodaltons after reduction (fig 2). The antibodies

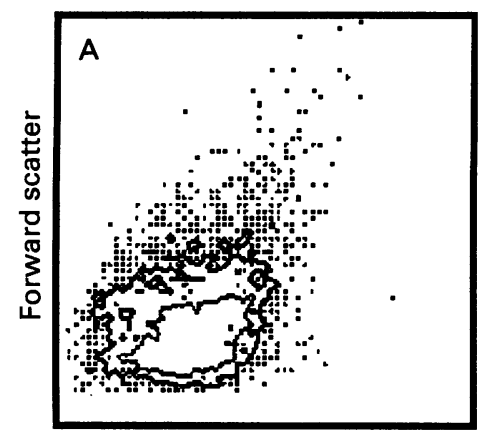

FITC-By44

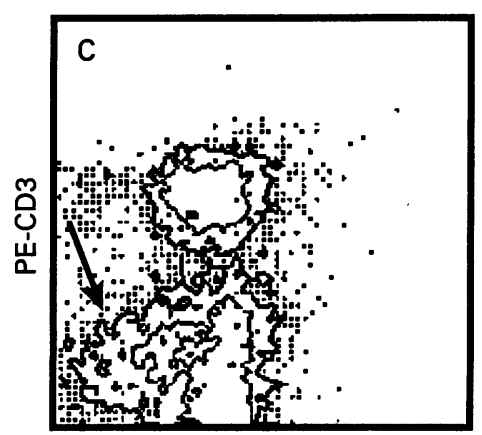

FITC-By44
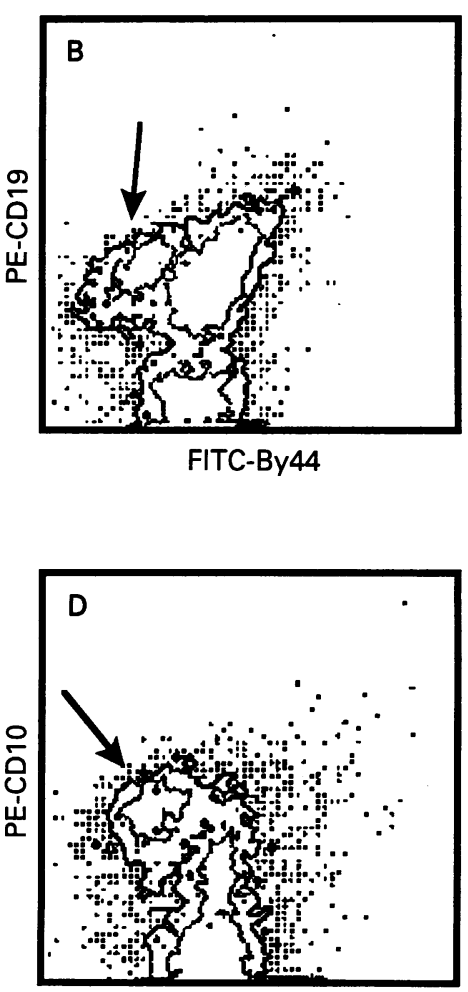

FITC-By44
Figure 3 Flow cytometric analysis of tonsil cells. (A) Single labelling with anti ICAM-3 shows that most cells (85\%) are positive. Double labelling studies to characterise the $I C A M-3$ negative cells shows that they are $C D 19$ positive $(B)$ and $C D 3$ negative (arrowed) (C). As they also express CD10 (arrowed) (D), they correspond to germinal centre B cells. In keeping with the tissue section staining, only about $50 \%$ of the germinal centre (CD10 positive) cells lack ICAM-3. also recognised an antigen of the same molecular weight on western blots of unreduced samples (data not shown).

\section{Results}

CELL SUSPENSIONS

All four antibodies labelled neutrophils, monocytes, and most (>98\%) lymphocytes in peripheral blood. In tonsil cell suspensions the antibodies labelled $85 \%$ of cells (the mean of five experiments). Double labelling studies of these cells showed that while all of the $\mathrm{T}$ cells (identified as $\mathrm{CD} 3$ positive cells) were also ICAM-3 positive, about $18 \%$ of $B$ cells (CD19 positive) were unlabelled by the ICAM-3 antibody. These ICAM-3 negative B cells appeared to be germinal centre cells as they also expressed CD10 (fig 3).

\section{TISSUES}

The expression of ICAM-3 by normal cells and tissues is summarised in table 1 . In the tonsil the antibodies showed extensive labelling of white cells, including mantle zone $B$ cells, plasma cells, and T cell areas. In germinal centres a proportion of the cells were unlabelled and the percentage of these negative cells varied considerably from one germinal centre to another (fig 1). Vascular endothelium did not stain, or rarely, stained very weakly. Epithelium was negative. These results were consistent with those obtained from the flow cytometry studies.

The staining of lymphoid tissue in the spleen was very similar to that of tonsil, but red pulp macrophages were also positive. In the thymus the antibodies labelled more than $90 \%$ of cells in the medulla with fewer cells staining in the cortex (about $80 \%$ of cells were labelled). In the liver Kupffer cells were labelled, as were scattered lymphocytes.

Apart from scattered leucocytes, no labelling was seen in non-haemopoietic tissues.

We compared the tissue distribution of ICAM-1 and 2 with ICAM-3, especially with regard to endothelial labelling (fig 1).

The pattern of ICAM-3 expression by cell lines is summarised in table 2.

The antibodies labelled neoplastic cells in most leukaemias and lymphomas, as summarised in table 3 . In a range of cases, including chronic lymphocytic leukaemia, hairy cell leukaemia, myeloma, immunoblastic lymphoma, and Hodgkin's disease, strong expression of ICAM-3 on vascular endothelium was also observed. This contrasted with the absent or weak staining of endothelium seen in reactive lymphoid tissue (fig 1). This staining was confined to the endothelium of small arteries, and in any one case only a proportion of these vessels were labelled. ReedSternberg cells and their variants in samples of Hodgkin's disease tissue were often stained negative, but because of the strongly positive staining adjacent reactive small lymphocytes, which were present around some of the ReedSternberg cells and their variants, it was impossible to state that they all remained unstained (fig 1). 
Table 1 Expression of ICAM-3 in normal cells and tissues

\begin{tabular}{|c|c|}
\hline Tissue & Labelling \\
\hline Peripheral blood & Monocytes, neutrophils and most (>98\%) of lymphocytes \\
\hline Tonsil & Mantle zones, plasma cells, $\mathrm{T}$ cells, and some germinal centre cells \\
\hline Spleen & Mantle and marginal zones, and some red pulp macrophages \\
\hline Thymus & Medulla and some cells in the cortex \\
\hline Liver & Kupffer cells and scattered white cells \\
\hline Lung & Scattered leucocytes \\
\hline Kidney & Scattered leucocytes and distal tubules \\
\hline Uterus & Scattered leucocytes \\
\hline Heart & Scattered leucocytes \\
\hline Muscle & Scattered leucocytes \\
\hline Pancreas & Scattered leucocytes \\
\hline Prostate & Scattered leucocytes \\
\hline Breast & Scattered leucocytes \\
\hline
\end{tabular}

Normal tonsil was obtained from the ENT Department of the Radcliffe Infirmary, Oxford. Other normal tissues obtained from the Histopathology Department of the John Radcliffe Hospital, Oxford.

\section{Discussion}

When used to study the pattern of expression of ICAM-3 on human cells, it was evident that this molecule has a novel pattern of distribution. It is constitutively expressed on most leucocytes, but a substantial minority of $B$ cells in germinal centres, and many lymphocytes in the thymic cortex, are ICAM-3 negative. This suggests that proliferation or activation of lymphoid cells may reduce the level of expression of ICAM-3. Most cases of non-Hodgkin's lymphoma that we tested were positive for ICAM-3, but in Hodgkin's disease the antigen was absent from ReedSternberg cells and their variants. This is in keeping with the idea that ICAM-3 is lost following cell activation, because these cells express CD30 which is one of the antigens associated with cell activation. We also found a small number $(<2 \%)$ of lymphoid cells in peripheral blood that were ICAM-3 negative. It may be that these lymphoid cells have lost surface ICAM-3 as a result of in vivo activation. This disappearance of ICAM-3 from lymphoid cells which are dividing or activated is illustrated by its absence from cells in the germinal centre of the tonsil.

ICAM-3 was essentially restricted to cells of haematopoietic origin. This was confirmed by staining cell lines, which revealed only one positive line out of four non-haematopoietic lines tested (KATO III, a gastric carcinoma), and in this exceptional sample only $5 \%$ of cells were reactive.

The results of Vazeux et $a l^{10}$ correlate well with our findings, although we could not confirm that ICAM-3 is present on lung epithelium.

The presence of ICAM-3 on resting lymphocytes and its absence from proliferating cells is in contrast to ICAM-1, which shows the reverse pattern. ${ }^{19}$ This complementary pattern was also true for endothelium, which was usually ICAM-1 positive, ICAM-3 negative. Although ICAM-3 was absent from, or only weakly expressed, on vascular endothelium in normal tissues, it was expressed strongly on endothelium in samples from a range of cases of lymphoid neoplasia, including chronic lymphocytic leukaemia, hairy cell leukaemia, myeloma and immunoblastic lymphoma. Both ICAM-1 and ICAM-2 are expressed more strongly on the high endothelial venules and other small vessels in malignant lymph nodes, ${ }^{20}$ and the endothelial expression of ICAM-3 in lymphoid neoplasia possibly occurs via a similar mechanism. It remains to be established whether these concomitant increases in expression of the three members of the ICAM family have implications in terms of altered leucocyte traffic.

We thank Dr TA Springer for his kind gift of antibodies RR1/1 and CBR-IC2/1.

This work was supported by grants from the Leukaemia Research Fund. H Turley is supported by the Imperial Research Fund. H Turley is supported by the Imperial Cancer Research Fund. I Doussis is

\footnotetext{
1 Springer TA. Adhesion receptors of the immune system Nature 1990;346:425-34.

2 Stoolman LM. Adhesion molecules controlling lymphocyte migration. Cell 1989;56:907-10.
}

3 Marlin SD, Springer TA. Purified intercellular adhesion

* Reactive lymphocytes in the cases of Hodgkin's disease were positive for ICAM-3 but the Reed

Leukaemic peripheral blood samples anticoagulated with heparin, from patients attending the John
Oxford, were used to prepare blood smears. 
molecule-1 (ICAM-1) is a ligand for lymphocyte function-associated antigen 1 (LFA-1). Cell 1987;51: 813-9.

4 Rothlein R, Dustin ML, Marlin SD, Springer TA. A human intercellular adhesion molecule (ICAM-1) A human intercelluar adhesion molecule (1CA.

5 Staunton DE, Dustin ML, Springer TA. Functional Staunton DE, Dustin ML, Springer TA. Functional
cloning of ICAM-2, a cell adhesion ligand for LFA-1 cloning of ICAM-2, a cell adhesion ligand for
homologous to ICAM-1 Nature 1989;339:61-4.

6 Nortamo P, Salcedo R, Timonen T, Patarroyo MM, Gahmberg CG. A monoclonal antibody to the human leukocyte adhesion molecule intercellular adhesion molecule-2. F Immunol 1991;146:2530-5.

7 de Fougerolles AR, Stacker SA, Schwarting R, Springer TA. Characterisation of ICAM-2 and evidence for a third counter-receptor for LFA-1. I Exp Med 1991; 174:253-67.

8 Akella R, Hall RE. Expression of the adhesion molecules ICAM-1 and ICAM-2 on tumor cell lines does not correlate with their susceptibility to natural killer cell-medi-
ated cytolysis: evidence for additional ligands for ated cytolysis: evidence for additional ligands for
effector cell $\beta_{2}$ integrins. Eur $\Im$ Immunol 1992;22: 1069-74.

9 Fawcett J, Holness CLL, Needham LA, et al. Molecular cloning of ICAM-3, a third ligand for LFA-1, constitutively expressed on resting leucocytes. Nature 1992;360:481-4.

10 Vazeux R, Hoffman PA, Tomita JK, et al. Cloning and characterization of a new intercellular adhesion molecule ICAM-R. Nature 1992;360:485-8.

11 Mason DY, Cordell J, Pulford KAF. Production of monoclonal antibodies for immunocytochemical use. In: monoclona antibodies for immunocytochemical use. In:
eds. Techniques in immunocytochemistry. Vol 2. New
York: Academic Press, 1983:175-216

12 Erber WN, Pinching AJ, Mason DY. Immunocytochemical detection of $T$ and $B$ cell populations in routine blood smears. Lancet 1984;i:1042-5.

13 Cordell $\mathrm{L}$, Falini B, Erber WN, et al. Immunoenzymatic labelling of monoclonal antibodies using immune complexes of alkaline phosphatase and monoclonal antplexes of alkaline phosphatase and monoclonal antalkaline phosphatase (APAAP

14 Mason DY, Abdulaziz Z, Falini B, Stein H. Single and double immunoenzymatic techniques for labelling tissue section with monoclonal antibodies. Ann N Y Acad Sci 1984;420:127-33.

15 Carter N. Introduction to the principles of flow cytometry. In: eds. Flow cytometry: A practical approach. Oxford: Oxford University Press, 1989:1-28.

16 Goding IW. Immunofluoresence. In: Monoclonal antibodies: principles and practice. New York: Academic Press, 1983:208-49.

17 Banerii J, Olson L, Schaffner W. A lymphocyte-specific cellular enhancer is located downstream of the joining region in immunoglobulin heavy chain genes. Cell 1983;33:729-40.

18 Laemmli UK. Cleavage of structural proteins during the assembly of the head of bacteriphage T4. Nature 1970;227:680-5.

19 de Fougerolles AR, Springer TA. Intercellular adehesion molecule 3, a third adhesion counter-receptor for lymphocyte function-associated molecule 1 on resting lymphocytes. F Exp Med 1992;175:185-90.

20 Renkonen R, Paavonen T, Nortamo P, Gahmberg CG Expression of endothelial adhesion molecules in vivo. Am ₹ Pathol 1992;140:763-7. 